\title{
A sextet of clusters in the Vela OB2 region revealed by Gaia $^{\star}$
}

\author{
Giacomo Beccari, ${ }^{1} \dagger$ Henri M.J. Boffin, ${ }^{1}$ Tereza Jerabkova, ${ }^{1,2,3}$ Nicholas J. Wright ${ }^{4}$, \\ Venu M. Kalari ${ }^{5}$, Giovanni Carraro ${ }^{6}$, Guido De Marchi ${ }^{7}$ and Willem-Jan de Wit ${ }^{8}$ \\ ${ }^{1}$ European Southern Observatory, Karl-Schwarzschild-Strasse 2, 85748 Garching bei München \\ ${ }^{2}$ Helmholtz Institut für Strahlen und Kernphysik, Universität Bonn, Nussallee 14-16, 53115 Bonn, Germany \\ ${ }^{3}$ Astronomical Institute, Charles University in Prague, V Holešovičkách 2, CZ-180 00 Praha 8, Czech Republic \\ ${ }^{4}$ Astrophysics Group, Keele University, Keele, ST5 5BG, UK \\ ${ }^{5}$ Departamento de Astronomia, Universidad de Chile, Casilla 36-D, Correo Central, Santiago, Chile \\ ${ }^{6}$ Dipartimento di Fisica e Astronomia Galileo Galilei, Vicolo Osservatorio 3, I-35122, Padova, Italy \\ ${ }^{7}$ Research 83 Scientific Support Department, ESA ESTEC, Keplerlaan 1, 2200 AG Noordwijk, The Netherlands \\ ${ }^{8}$ European Southern Observatory, Alonso de Córdova 310\%, Casilla 19001, Santiago, Chile
}

Accepted XXX. Received YYY; in original form ZZZ

\begin{abstract}
Using Gaia DR2 data, combined with OmegaCAM ground-based optical photometry from the ADHOC survey, and detailed Radial Velocity measurements from ESO-Gaia, we analyse in detail a $10 \times 5$ deg region around the Wolf-Rayet star $\gamma^{2}$ Vel, including the previously known clusters Gamma Vel and NGC2547. Using clustering analysis that considers positions, proper motions and parallax, we discover 6 clusters or associations -4 of which appear new. Analysis of the colour-magnitude diagram for these clusters show that 4 of them formed coevally from the same molecular clouds 10 Myr ago, while NGC 2547 formed together with a newly discovered cluster 30 Myr ago. This study shows the incredible wealth of data provided by Gaia for the study of young stellar clusters.
\end{abstract}

Key words: Stars: formation - Stars: pre-main sequence - Open clusters and associations

\section{INTRODUCTION}

Young clusters are a formidable tool to study star formation, stellar evolution, binary stars, as well as the formation and evolution of clusters themselves. They thus offer a window to observationally answer questions in many astrophysical domains. Hence, it is critical to discover and study as wide a range of clusters as possible.

We recently concluded the Accretion Disk with OmegaCAM (ADHOC) survey on the VLT Survey Telescope (VST), whose main goal is to study the population of Pre-Main sequence stars identified using $\mathrm{H} \alpha$ excess emission as signature of on-going accretion. As part of the survey, we observed a $10 \times 5$ deg region around the Wolf-Rayet Star $\gamma^{2}$ Vel. This region has received considerable attention in the recent years after the study by Jeffries et al. (2014) which, using Radial Velocity (RV) measurements from the Gaia-ESO survey (GES Gilmore et al. 2012; Randich et al.

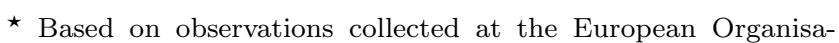
tion for Astronomical Research in the Southern Hemisphere under ESO programme(s) 188.B-3002, 096.C-0730(A) and 097.C0749(A)

$\dagger$ E-mail: gbeccari@eso.org
2013) found that the cluster around $\gamma^{2}$ Vel (namely Gamma Vel) is in fact composed of two coeval but kinematically distinct populations A and B. Interestingly Sacco et al. (2015) used GES observations to study the stellar population on a region centered on NGC 2547 , a $\approx 35$ Myr cluster located two degrees south of $\gamma^{2}$ Vel. They find a population of stars whose RVs, lithium absorption lines, and positions in a colour-magnitude diagram (CMD) are consistent with those of Gamma Vel B, which they call NGC 2547 B. It is hence likely that the stars observed by Jeffries et al. (2014, Gamma Vel B) and Sacco et al. (2015, NGC 2547 B) in fact belong to a young, low-mass stellar population spread over at least several square degrees in the Vela OB2 complex. Such result has been supported via a set of N-BODY simulation from Mapelli et al. (2015) and might imply that stars in NGC 2547 B could have originally been part of a cluster around $\gamma^{2}$ Velorum that expanded after gas expulsion or formed in a less dense environment that is spread over the whole Vela OB2 region (Prisinzano et al. 2016). More recently Damiani et al. (2017) used the GAIA DR1 catalogue TGAS to perform a proper-motion (PM) study of the stellar population in a radius of 4 degrees from the midpoint between the $\gamma^{2} \mathrm{Vel}$ and the NGC 2547 center. They find 2 clear and well distinguished stellar populations 
in the PM space offered by TGAS namely $\mathrm{C}$ and D. Using GAIA-G and 2MASS Ks band photometry, they suggest that population-D is older than $\mathrm{C}$. Hence, population D is likely consistent with NGC 2547 while population C seems to belong to the Gamma Velorum cluster. Still, no firm conclusion could be drawn based on the GAIA DR1 data. Armstrong et al. (2018) traced the spatial distribution of low-mass stars across Vela OB2 using Gaia DR1 photometry and identified a number of spatial overdensities hinting at an extended low-mass population in the area.

The recent second Gaia data release (DR2 Gaia Collaboration et al. 2018) offers an unique opportunity to characterise and discover new stellar clusters thank to the very precise parallaxes and PMs. Here, we use Gaia DR2 data, combined with new ground-based optical photometry to study a wide region around $\gamma^{2}$ Velorum and determine precise properties of several clusters or associations therein.

\section{PHOTOMETRIC AND ASTROMETRIC DATA}

We secured with OmegaCAM, attached to the 2.4-m VST telescope in Paranal, a set of deep multi-exposures of a region covering $126<\alpha<116 \mathrm{deg}$. and $-50<\delta<-44.5 \mathrm{deg}$. Data were obtained with the $u, g, r, i$ and $H \alpha$ filters, although we will here use mostly the images obtained with the $r$ - and $i$ filters. We acquired $2 \times 25$ s exposures for each pointing in the $r$ and $i$ band. Data reduction was carried out at the Cambridge Astronomical Survey Unit (CASU) and we downloaded the astrometrically and photometrically calibrated single band catalogs from the VST archive at CASU ${ }^{1}$. We used a large number of stars in common with the AAVSO Photometric All-Sky Survey (APASS) to correct for possible residual offsets in $r$ and $i$ magnitudes between each single exposures. The final catalogue includes $1,847,804$ objects homogeneously sampled in $r$ and $i$ bands down to $r \approx 21.5 \mathrm{mag}$ in ABMAG.

We retrieved from the Gaia science archive $^{2}$ all the objects detected by Gaia that are within 10 degrees on the sky from $\alpha \approx 120.4$ deg., $\delta \approx-47.5 \mathrm{deg}$. without any additional filtering. We then used the $C^{3}$ python script from (Riccio et al. 2017) in order to identify the stars in common between the OmegaCAM and the Gaia catalogue. We found 1,785,477 targets in common between Gaia and OmegaCAM. We note here that all stars with magnitude $r>20$ are lost in this process because of the detection limit of the GAIA DR2 catalogue. Finally the GES provides us with RVs for 1369 targets in our catalogue.

\section{FINDING CLUSTERS}

The data-set that we have collected so far is ideal to investigate the stellar population in the Vela OB2 region. On one side, the Gaia DR2 in fact provides for all the stars in the sampled region, 5 parameter-solutions $\alpha, \delta$, PMs in $\alpha$ and $\delta$ $\left(\mu_{\alpha}\right.$ and $\mu_{\delta}$ ), as well as the parallax, $\varpi$. On the other hand,

1 http://casu.ast.cam.ac.uk/vstsp/

2 http://gea.esac.esa.int/archive/ the OmegaCAM data-set provides accurate multi-band photometric measurements. These data can hence be used to identify sub-populations via clustering algorithms and to study their properties (e.g. age) through the CMD. We want to stress here that in this work we are mainly interested in finding groups of stars whose astrometric and photometric properties allow us to infer their membership to a specific sub-population or association in the studied field, and we are not aiming at completeness. In this respect, we will use the term cluster to indicate a population of stars showing common astrometric and photometric properties. We will study the dynamical state of the sub-populations to assess their dynamical state (e.g. virial status) in a follow-up work.

\subsection{In position and parallax}

To find clusters, we use the python implementation in sci-kit learn $^{3}$ (Pedregosa et al. 2011), of the DBSCAN data clustering algorithm (Ester et al. 1996). As a first step, we select only those stars that have a relative error on the parallax smaller than $10 \%$, i.e. $\sigma_{\varpi} / \varpi<0.1$. Since we are mostly interested in clusters located around the star forming region surrounding $\gamma^{2} \mathrm{Vel}$, we made a cut in parallax, $2.2<$ $\varpi<3.9 \mathrm{mas} / \mathrm{y}$, as $\gamma^{2} \mathrm{Vel}$ has a parallax of $2.92 \pm 0.30 \mathrm{mas} / \mathrm{y}$ (van Leeuwen 2007). The stars in the final catalogue show an average value of visibility_periods_used $=15$. Using equation $(1)^{4}$ from Arenou et al. (2018) and filtering for duplicate targets, we remove only $3 \%$ of stars. We do not consider any photometric filter introduced by Arenou et al. (2018) as we use the OmegaCAM photometry.

First, we were interested in looking for clusters in the sky position, and we thus applied DBSCAN in the 3D space, $(\alpha, \delta, \varpi)$, rescaling these three sets of data such that they all had a mean of zero and a standard deviation of 1 , and used a scale of 0.25 and a minimum sample of 20 . We note here that we have checked that we obtain similar results when using a different value for the minimum sample, and adjust accordingly the scale factor. The clustering method allowed us to distinguish 5 sub-populations on sky (see top left panel of Fig. 1), one of which (Cl5) is however very poorly populated. We show in the bottom panel on the left-hand of Fig. 1, the PMs of the stars belonging to the five clusters discovered in $\alpha$ and $\delta$. Clearly, four of the sub-groups also appear clustered in PM space and are thus indeed kinematically compact. The arrows shown in some of the panels indicate the mean motion in the plane of the sky and are centred on the mean position of the sub-group. Quite amazingly, three of the clusters ( $\mathrm{Cl} \mathrm{1,2}$ and 4$)$ have very similar PMs. The smallest concentration we found $(\mathrm{Cl} 5)$ contains only 9 stars but several of them have also similar PMs as $\mathrm{Cl} 4$. Clusters 1, 2,4 , and 5 are also evidence on the density map of low-mass stars in Vela OB2 identified by Armstrong et al. (2018).

\footnotetext{
3 http://scikit-learn.org/stable/modules/ generated/sklearn.cluster.DBSCAN.html

${ }^{4}$ Equation (1) presents $\boldsymbol{u}$-parameter that, combining the astrometric_chi2_al and astrometric_n_good_obs_al parameters, ensures that we select only the targets with good astrometric solution.
} 

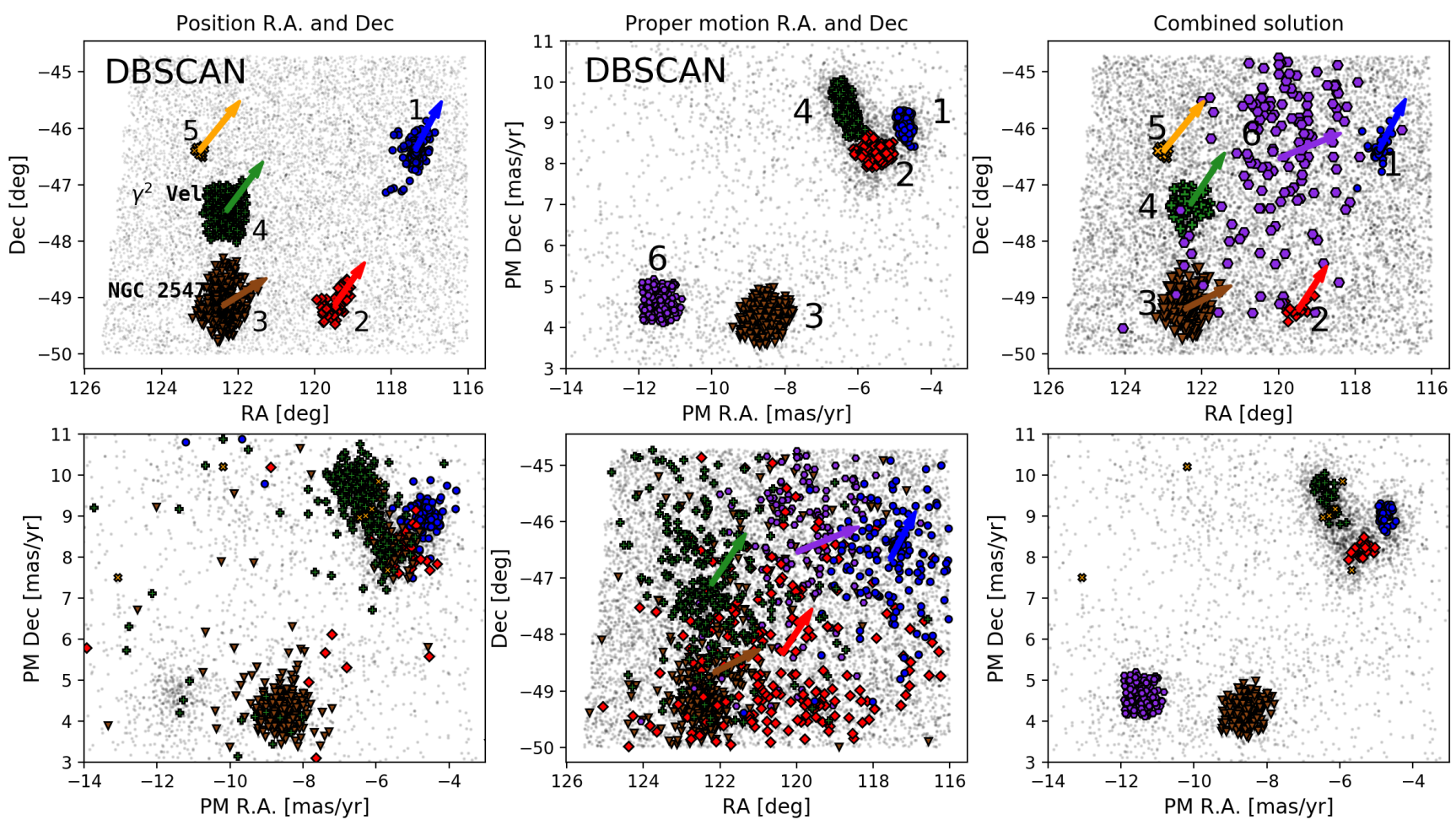

Figure 1. Left-column: (top) Cluster selection in the $(\alpha=$ R.A., $\delta=$ Dec) space with the arrow indicating the mean motion in the plane of the sky and centred on the mean position of the cluster. The same stars are also shown in the proper motion space (bottom-left). Cluster 1 is shown in blue, 2 in red, 3 in brown, 4 in green and 5 in orange. Central-column: (top) Cluster selection in the ( $\mu_{\alpha}=\mathrm{PM}$ R.A, $\mu_{\delta}=\mathrm{PM}$ Dec) space. The new cluster 6 is shown in violet. The same stars are shown in the $\alpha / \delta$ plan (bottom). Central-column: Location in $\alpha$ and $\delta$ (top) and in PM R.A and PM Dec (bottom) of the stars which are in common between the clustering selections applied in the previous plots.

Table 1. Mean properties of the six clusters.

\begin{tabular}{|c|c|c|c|c|c|c|c|c|c|}
\hline Cl. ID & $\begin{array}{c}\alpha \\
\text { (deg.) }\end{array}$ & $\begin{array}{c}\delta \\
\text { (deg.) }\end{array}$ & $\begin{array}{c}\mathrm{PM} \alpha \\
(\mathrm{mas} / \mathrm{yr})\end{array}$ & $\begin{array}{c}\text { PM } \delta \\
(\mathrm{mas} / \mathrm{yr})\end{array}$ & $\begin{array}{l}\text { Parallax } \\
\text { (mas) }\end{array}$ & $\begin{array}{l}\text { Distance } \\
\quad \text { (pc) }\end{array}$ & $\begin{array}{c}\text { Rad. Vel. } \\
(\mathrm{km} / \mathrm{s})\end{array}$ & $\begin{array}{l}\text { Age } \\
\text { Myr }\end{array}$ & Other Name \\
\hline 1 & 117.366448 & -46.339979 & $-4.76 \pm 0.12$ & $9.05 \pm 0.17$ & $2.52 \pm 0.04$ & 397 & - & 10 & \\
\hline 2 & 119.566307 & -49.212074 & $-5.41 \pm 0.19$ & $8.18 \pm 0.15$ & $2.42 \pm 0.05$ & 413 & $20.36 \pm 0.06$ & 10 & \\
\hline 3 & 122.468165 & -49.176393 & $-8.57 \pm 0.29$ & $4.27 \pm 0.27$ & $2.54 \pm 0.07$ & 393 & $12.29 \pm 3.71$ & 30 & NGC 2547 \\
\hline 4 & 122.343657 & -47.350962 & $-6.40 \pm 0.20$ & $9.56 \pm 0.32$ & $2.85 \pm 0.08$ & 351 & $18.50 \pm 4.50$ & 10 & Gamma Vel \\
\hline 5 & 123.013175 & -46.400231 & $-7.52 \pm 2.36$ & $9.22 \pm 1.17$ & $2.74 \pm 0.05$ & 365 & - & 10 & \\
\hline 6 & 119.999214 & -46.525013 & $-11.47 \pm 0.26$ & $4.65 \pm 0.27$ & $3.02 \pm 0.07$ & 331 & $11.41 \pm 4.20$ & 30 & \\
\hline
\end{tabular}

\subsection{In proper motions}

The inspection of the bottom panel on the left-hand of Fig. 1 clearly reveals that there is a cluster in PM space that was not caught via the selection in $\alpha$ and $\delta$ (see small group in the lower left). We thus also looked at clustering in this space, by running DBSCAN in the $\left(\mu_{\alpha}, \mu_{\delta}, \varpi\right)$ space. Here also five clusters are clearly identified (see top panel in the central column of Fig. 1), four of which are in common with the ones found with the previous method. Interestingly, while $\mathrm{Cl}$ 5 is not found in PM, as expected, a new cluster ( $\mathrm{Cl} \mathrm{6)}$ was found at $\mu_{\alpha} \approx-11.5 \mathrm{mas} / \mathrm{y}, \mu_{\delta} \approx 4.6 \mathrm{mas} / \mathrm{y}$, that corresponds to stars more loosely spread on the sky. We show in the lower panel of the central column of the figure, the position in $\alpha$ and $\delta$ of the stars selected in PM space. The fact that some clusters are found in PM space but not in $\alpha / \delta$ space (and vice versa) clearly demonstrates that we should not be looking at clusters in 5-dimensional space (positions, PMs, parallaxes) only, but that the spatial information should be consistently checked.

As final step in our study, we have then taken a conservative approach and retained, for the 4 clusters where this applies (namely $\mathrm{Cl} \mathrm{1,2,3}$ and 4), only those stars that are selected using both methods. This allows us to have a much better indication on the location and motion of the clusters, at the expense of its completeness. We show in the righthand column of Fig. 1 the position of the final selection of sub-groups in the $\alpha / \delta$ (top right plot) and PM space (bottom right plot). We list in Table 1 the mean properties for the final set of clusters identified with the combination of the two approaches. 

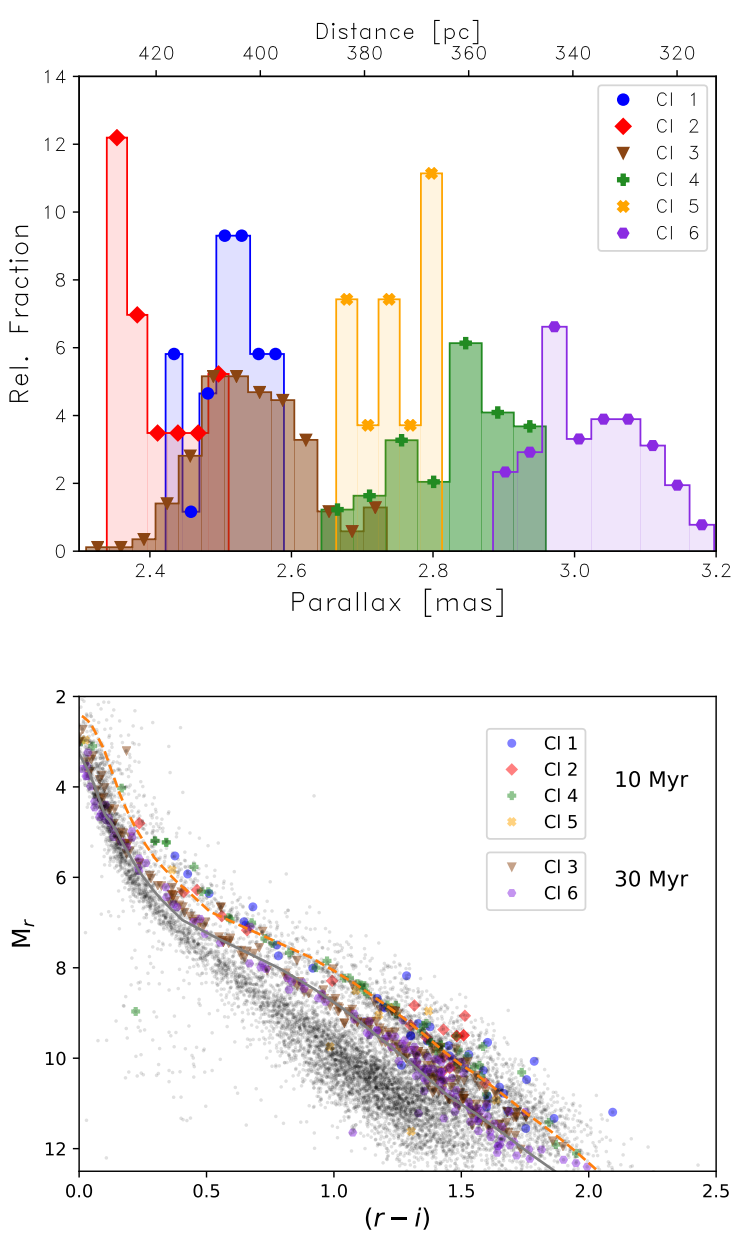

Figure 2. (top) The parallax distribution of the 6 clusters discussed in this paper. The distributions have been normalised. (bottom) The colour-magnitude $(r-i)-M_{r}$ diagram for all stars in the field with $2.2<\varpi<3.9$ and $\sigma_{\varpi} / \varpi<0.1$ (black dots) and the stars of the clusters. In all plots, the colours correspond to those shown in Fig. 1.

\section{PROPERTIES OF THE CLUSTERS}

We will discuss from now on, only the stars belonging to the sub-populations (or clusters) as shown in the plots in the right-hand column of Fig. 1. It is immediately obvious that $\mathrm{Cl} 3$ and 4 coincide with the well known clusters NGC 2547 and Gamma Vel while the position of the other clusters does not correspond to any known cluster. In Fig. 2, we show the parallax distribution and the CMD of the 6 clusters. Given the fact that the clustering was made in a $3 \mathrm{D}$ space that included the parallax, it is no surprise that each cluster corresponds to a well defined (and limited) parallax range. Given that we only use those stars which have precise parallaxes, we can simply infer the distances from these, without needing any prior knowledge on the Bayesian probabilities of our targets. It is seen that 3 clusters $(\mathrm{Cl} 1$, $2,3)$ are at similar distances $(\approx 400 \mathrm{pc})$, while the 3 others are closer to us (between 330 and $360 \mathrm{pc}$ ). The CMD reveals the incredible precision of the OmegaCAM photometry and Gaia parallaxes and very well defined sequences, indicative of very young populations (in comparison to the background of galactic stars). The $\mathrm{H} \alpha$ photometry confirms that very few stars show $\mathrm{H} \alpha$ excess, usually used as sign of on-going accretion in pre-Main Sequence stars (e.g. Kalari et al. 2015). This is in agreement with the fact that stars in the Vela OB2 region are older then $10 \mathrm{Myr}$ and, hence, no accretion discs are expected (Fedele et al. 2010). The age for each cluster, obtained using solar metallicity models (Spina et al. 2014) from Bressan et al. (2012) and $A_{V}=0.18$ (in agreement with Sacco et al. 2015), are listed in Tab. 1. The fit of the CMD for each cluster is shown in Fig. 2.

\section{DISCUSSION}

Jeffries et al. (2014) find 2 kinematically distinguished populations around $\gamma^{2}$ Velorum, an old (10 Myr), bound $\left(\sigma_{A}=\right.$ $\left.0.34 \pm 0.16 \mathrm{kms}^{-1}\right)$ population A with a mean $\mathrm{RV}_{A}=16.73 \pm$ $0.09 \mathrm{kms}^{-1}$ and centrally concentrated around $\gamma^{2}$ Velorum. Population B is more dispersed, slightly younger and has a broader dispersion of $1.60 \pm 0.37 \mathrm{kms}^{-1}$ and is offset in $\mathrm{RV}$ from the first by $2 \mathrm{kms}^{-1}$. Jeffries et al. (2014) propose several scenarios to explain the presence of two populations, concluding that population $\mathrm{A}$ is the remnant of a bound cluster formed around the massive binary, and population B is a dispersed population from the wider Vela OB2 association. Later, Sacco et al. (2015) in a GES-based RV study of the stellar population in the cluster NGC 2547, discovered 15 stars that appear kinematically similar to population B of Gamma Vel and are located $2 \mathrm{deg}$ south of $\gamma^{2}$ Velorum.

Thanks to the combination of GAIA DR2, OmegaCAM photometry and the GES measurements, we are able now to investigate with greater precision the stellar populations in the area. By combining parallaxes, PMs and radial distribution of the stars in the area, we identify 6 different clusters. According to our study, NGC 2547 (Cl3) is located at a distance of $393 \mathrm{pc}$ (hence larger than the distance of 340pc adopted by Sacco et al. 2015), shows an age of $30 \mathrm{Myr}, \mu_{\alpha}=-8.57 \pm 0.29 \mathrm{mas} / \mathrm{y}$ and $\mu_{\delta}=4.27 \pm 0.27 \mathrm{mas} / \mathrm{y}$ and $\mathrm{RV}=12.29 \pm 3.71 \mathrm{kms}^{-1}$ in agreement with the population of NGC 2547 A from Sacco et al. (2015). The main cluster around $\gamma^{2}$ Velorum, (Gamma Vel A/population A in Jeffries et al. 2014) corresponds to our Cl4. It shows a distance of $351 \mathrm{pc}$, age of $10 \mathrm{Myr}$ (older than the estimated age of the Wolf-Rayet star $\approx 5.5 \pm 1 \mathrm{Myr}$; Eldridge 2009), $\mu_{\alpha}=-6.40 \pm 0.20 \mathrm{mas} / \mathrm{y}$ and $\mu_{\delta}=9.56 \pm 0.32 \mathrm{mas} / \mathrm{y}$ and $\mathrm{RV}=18.50 \pm 4.50 \mathrm{kms}^{-1}$. Hence, the main population of the $\mathrm{Cl} 3$ (NGC2547 A) and Cl4 (Gamma Vel A) clusters are separated by $\approx 44 \mathrm{pc}$ in 3 -D. We emphasise here that thanks to the parallaxes and PMs information available with GAIA DR2, we could naturally remove any contamination from a population "B" in the RV distribution of both the clusters NGC 2547 and Gamma Vel. Noticeably, Cl2 (clearly identified both in $\mathrm{PM}$ and $\mathrm{RA} / \delta$ space) has properties in term of PMs and age similar to $\mathrm{Cl} 4$ (Gamma Vel A), but it is located at a larger distance (413 pc). RV measurements from GES are available for some of the stars belonging to $\mathrm{Cl} 2$ and selected in the PM space. These stars show an average $\mathrm{RV}=20.36 \pm 0.6 \mathrm{kms}^{-1}$, which is in good agreement with NGC 2547B studied by (Sacco et al. 2015). Cl2 has a well-defined centre in the $\alpha$ and $\delta$ space and is located at a 3-D distance of 64 pc from Gamma Vel and 24 pc from NGC 2547. It is likely that such stars were identified as NGC 2547 B by Sacco et al. (2015). Our data seems to indicate that they 
do not belong to Gamma Vel even if they show the same age as they are located at a much larger distance.

Cluster 6 (violet) is very interesting as it appears predominantly in the PM space, but is much looser in the apparent sky position. It is, however, very well constraint in parallax and located at a distance of $\approx 330 \mathrm{pc}$. Most interesting is that it has an age, RV and PM properties which are similar to that of $\mathrm{Cl} 3$, i.e. NGC 2547, although its centre is located 65 pc away from this cluster in 3-D.

Hence, to summarise, $\mathrm{Cl} 2$ and $\mathrm{Cl} 4$ (Gamma Vel) show same age and PM properties but a difference of RV of $\approx 6 \mathrm{kms}^{-1}$ and a 3 -D distance of $64 \mathrm{pc}$ from each other. Similarly Cl6 and Cl3 (NGC 2547) do show same age and similar $\mathrm{PM}$ and $\mathrm{RV}\left(\approx 12 \mathrm{kms}^{-1}\right)$ but are located at a $3-\mathrm{D}$ distance of $65 \mathrm{pc}$ from each other. Cl1 also show PM and age similar to Cl4 (Gamma Vel) but is located at a 3-D distance of $52 \mathrm{pc}$ from it and $31 \mathrm{pc}$ from the much older $\mathrm{Cl} 3$ (NGC 2547). Interestingly, it is located at a 3D distance of $28 \mathrm{pc}$ from the coeval $\mathrm{Cl} 2$ which show similar position in the PM space. No RV information are available for Cl1.

Overall, our study put the stellar populations in the Vela OB2 complex under a new prospective. Thanks to the unique capabilities offered by the Gaia-Dr2 catalogues, it is indeed proved that the stellar " $\mathrm{B}$ " populations originally discovered in Gamma Vel and NGC 2547 by Jeffries et al. (2014) and Sacco et al. (2015) respectively, are in fact stars belonging to a complex set of clusters overlapping in space over an area of almost 100 pc. The RV information available for each cluster, combined with the PMs, indicate that all the clusters move on an almost parallel trajectory from SouthWest to North-East. Based on the available data and tracing back the trajectory of the stars for which RV and PMs are available, we could not find a common location from where the clusters could have originated 10 to $30 \mathrm{Myr}$ ago.

It is interesting to note that $\mathrm{OB}$ associations show spatial and kinematic substructure in the form of groups and clumps that potentially will survive as the entire association disperses. In this context, the initial conditions of the complex of clusters found in this work are likely to be very similar to that seen (structurally and kinematically) in younger OB associations such as Cygnus OB2 (Wright et al. 2016) and Scorpius- Centaurus (Wright \& Mamajek 2018). It is hence reasonable to hypothesize that such regions might resemble the progenitor of the distribution of Vela OB2 comoving clusters at 5-10 Myr time. In more distant star forming regions, particularly outside the Milky Way, without accurate PM information clusters of this type would be hard to distinguish from one another. This could explain the spread of ages observed e.g. in young star forming regions in the Magellanic Clouds (De Marchi et al. 2017). While we can not discard that the clusters are in fact sub-clusters formed inside the same molecular clouds in a fragmented and filamentary structure, more detailed kinematic information will be needed to re-construct the star formation history of this complex region in order to firmly confirm if, where and when the stars in these clusters formed.

\section{ACKNOWLEDGEMENTS}

This research has made use of the services of the ESO Science Archive Facility. This work has made use of data from the European Space Agency (ESA) mission Gaia (https://www.cosmos.esa.int/gaia), processed by the Gaia Data Processing and Analysis Consortium (DPAC, https://www. cosmos.esa.int/web/gaia/dpac/consortium). Funding for the DPAC has been provided by national institutions, in particular the institutions participating in the Gaia Multilateral Agreement. Based on data products from observations made with European Southern Observatory Telescopes at the La Silla Paranal Observatory under programme ID 188.B-3002. These data products have been processed by the Cambridge Astronomy Survey Unit (CASU) at the Institute of Astronomy, University of Cambridge, and by the FLAMES/UVES reduction team at INAF/Osservatorio Astrofisico di Arcetri.

\section{REFERENCES}

Arenou F., et al., 2018, preprint, (arXiv:1804.09375)

Armstrong J. J., Wright N. J., Jeffries R. D., 2018, MNRAS

Bressan A., Marigo P., Girardi L., Salasnich B., Dal Cero C., Rubele S., Nanni A., 2012, MNRAS, 427, 127

Damiani F., Prisinzano L., Jeffries R. D., Sacco G. G., Randich S., Micela G., 2017, A\&A, 602, L1

De Marchi G., Panagia N., Beccari G., 2017, ApJ, 846, 110

Eldridge J. J., 2009, MNRAS, 400, L20

Ester M., Kriegel H.-P., Sander J., Xu X., 1996. AAAI Press, pp 226-231

Fedele D., van den Ancker M. E., Henning T., Jayawardhana R., Oliveira J. M., 2010, A\&A, 510, A72

Gaia Collaboration Brown A. G. A., Vallenari A., Prusti T., de Bruijne J. H. J., Babusiaux C., Bailer-Jones C. A. L., 2018, preprint, (arXiv: 1804.09365)

Gilmore G., et al., 2012, The Messenger, 147, 25

Jeffries R. D., et al., 2014, A\&A, 563, A94

Kalari V. M., et al., 2015, MNRAS, 453, 1026

Mapelli M., et al., 2015, A\&A, 578, A35

Pedregosa F., et al., 2011, Journal of Machine Learning Research, 12,2825

Prisinzano L., et al., 2016, A\&A, 589, A70

Randich S., Gilmore G., Gaia-ESO Consortium 2013, The Messenger, 154, 47

Riccio G., Brescia M., Cavuoti S., Mercurio A., di Giorgio A. M., Molinari S., 2017, PASP, 129, 024005

Sacco G. G., et al., 2015, A\&A, 574, L7

Spina L., et al., 2014, A\&A, 567, A55

Wright N. J., Mamajek E. E., 2018, MNRAS, 476, 381

Wright N. J., Bouy H., Drew J. E., Sarro L. M., Bertin E., Cuillandre J.-C., Barrado D., 2016, MNRAS, 460, 2593

van Leeuwen F., 2007, A\&A, 474, 653 